"The submitted manuscript has been authored by a contractor of the U.S. Government under contract No. DE-AC05-96OR22464. Accordingly, the U.S. Government retains a nonexclusive royalty-free license to publish or reproduce the published form of this contribution, or allow others to do so, for U.S. Government purposes."

\author{
CONF- $980.560--$
}

\title{
Noble gas impurity balance and exhaust model for DIII-D and JET*
}

D.L. Hillis ${ }^{1}$, J. Hogan ${ }^{1}$, M. von Hellermann ${ }^{2}$, J. Ehrenberg ${ }^{2}$, L. Horton ${ }^{2}$, R. König ${ }^{2}$, P. Morgan ${ }^{2}$, G. Saibene ${ }^{2}$, and M.R. Wade ${ }^{1}$

IOak Ridge National Laboratory, Oak Ridge, TN 37831, USA

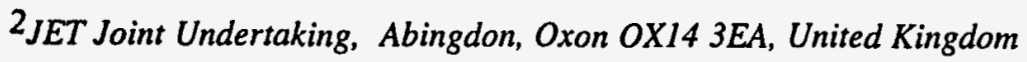

\section{Abstract}

Experiments to study the exhaust of noble gases (helium, neon) with cryopumping in DIIID [Advanced Divertor Program (ADP) configuration] and in JET (Mk1 configuration) found significant differences in the global exhaust rate of helium, while efficient neon exhaust was observed in both machines. An attempt to better understand the basic processes governing the exhaust of noble gases in ELMy H-mode with cryopumping has been undertaken. Since divertor geometries investigated in the DIII-D and in the JET cases have significant differences, a comparative modeling study has been undertaken using the MIST core impurity transport code and the b2.5 time-independent divertor transport code. Photodiode measurements are used to determine ELM frequency, and charge-exchange recombination (CER) measurements are compared with the MIST ELM model to evaluate transport coefficients in the core plasma. A significant reduction in the anomalous diffusivities is found for the non-ELM component of radial transport without the need for a pinch velocity, and the model provides a more coherent description than the conventional ELM-averaged approach. Sensitivity to boundary conditions has been studied through establishment of a database of divertor enrichment cases using b2.5.

Keywords: DIII-D, JET, tokamak, ELMs, helium pumping, neon, transport

*Research sponsored in part by the U.S. Department of Energy, under contract number DE-AC05-960R22464 with Lockheed Martin Energy Research Corporation.

DistRigution of this DOCUMENT is unLMTED $f_{1}$ 


\section{DISCLAIMER}

This report was prepared as an account of work sponsored by an agency of the United States Government. Neither the United States Government nor any agency thereof, nor any of their employees, makes any warranty, express or implied, or assumes any legal liability or responsibility for the accuracy, completeness, or usefulness of any information, apparatus, product, or process disclosed, or represents that its use would not infringe privately owned rights. Reference herein to any specific commercial product, process, or service by trade name, trademark, manufacturer, or otherwise does not necessarily constitute or imply its endorsement, recommendation, or favoring by the United States Government or any agency thereof. The views and opinions of authors expressed herein do not necessarily state or reflect those of the United States Government or any agency thereof. 


\section{Introduction}

The particle balance for noble gases in ELMy H-mode is of fundamental interest because of the need to exhaust helium in a reactor and of operational interest since injection of nonreacting gases (neon, argon, krypton) is proposed to produce a highly radiative divertor and thereby reduce the heat flux to the graphite divertor tiles. The suitability of H-mode confinement for reactors relies on the observed beneficial effect of ELMs in maintaining quasi-steady densities of both the working gas and the impurities. While ELMy H-mode energy and particle confinement have been found to be compatible with next-step reactor requirements in many machines, (e.g., ITER) [1], recent observation of greatly enhanced heat fluxes during type I ELMs raises doubts $[2,3]$. Thus, a better quantitative description of the trade-off between the ELM amplitude needed for effective particle exhaust and a tolerable maximum heat flux is required.

Previous experiments on the exhaust of helium and neon with cryopumping in DII-D [Lower Single Null (LSN) ADP configuration] and in JET (LSN Mk1 configuration) found significant differences in the global exhaust rate of helium, while efficient neon exhaust was observed in both machines [1, 4-6]. Experiments were done in H-mode with 'giant', or 'type-I', ELMs. Estimates [7] show that helium ions must be removed within 7 to 15 energy confinement times to maintain continuous reactor operation ( $\tau_{H e}^{*} / \tau_{E} \approx 7-15$ ). Utilizing argon frost pumping (a 10 micron argon frost layer) of helium on DII-D a value of $\tau_{H_{e}}^{*} / \tau_{E} \approx 8$ [4] was achieved, which is encouraging for a future fusion reactor. Utilizing a similar 10 micron argon frost layer on JET in the Mk1 divertor, a value of $\tau_{H e}^{*} / \tau_{E} \approx 15-20$ was obtained [5]. The observed differences for helium exhaust were ascribed to differences in the application of the argon frost technique [8] and to the injection of deuterium and helium to deduce the helium pumping speed prior to the JET experiments. This substantially reduced the helium pumping and spoiled/poisoned" the argon frost layer for providing high efficiency helium pumping. Neon is readily pumped by the cryopumps of both the JET and DIII-D pumping systems and adequate pumping was observed in both cases. Subsequent experiments with $D_{2}$ injection in DII-D have shown that neon divertor enrichment is greater than that of helium, and that both can be enhanced with an induced $\mathrm{D}^{+}$flow in the scrape-off layer [9].

This paper examines the core, scrape-off layer and divertor processes governing helium and neon exhaust to identify similarities and differences. Since the divertor geometries in the cases studied (DIII-D ADP and JET Mk1) are significantly different, a comparative modeling study has been employed to relate exhaust processes in these two experiments. The processes considered are: ELM effects on core transport and enrichment, relative effect of wall reflection coefficients for helium and neon in providing effective exhaust, and device-specific exhaust effects: argon-frost poisoning (for JET Mk1). The modeling components are: time-dependent MIST core radial 
impurity transport code [10], the b2.5 divertor code [11,12], and the B2-Eirene code system $[13,14]$. Since these tools have been employed previously for the DIII-D cases, the bulk of the analysis presented here is devoted to analyzing the JET Mk1 results in the same way. Attention is drawn to previous analysis of these processes in JET discharges using EDGE2D, CHEAPSANCO, NIMBUS, and other codes $[15,16]$.

\section{ELM effects on core transport}

In the MIST ELM model $[9,17]$, an ELM event is modeled as the expulsion of a specified fraction of all the $k$ charge-state densities, $A_{E L M} n_{k}(\rho, t)$, for a normalized radius $\rho>\rho E L M$. AELM is the expulsion fraction, PELM is the radius where ELMs affect the particle transport, and $\mathrm{n}_{\mathrm{k}}(\rho, \mathrm{t})$ is the density of the impurity species. This approach differs from previous models $[18,19]$ in that the ELM is treated as an instantaneous MHD instability, rather than as an extended period of high (L-mode or hybrid) diffusivity. An extended period of high recycling does occur in this model, but with a duration and magnitude governed by the system exhaust and quiescent transport parameters, rather than by an ad-hoc enhanced ELM diffusivity. The times of ELM events (frequency, $f_{E L M}$ ) are determined by the measured time histories of the $D_{\alpha}$ signals. The expulsion fraction is not determined by the $D_{\alpha}$ signal, and thus both AELM and PELM become fitting parameters, replacing the ad hoc time dependent variation in the anomalous diffusivity, $D_{A}$, and in the pinch velocity, $V_{\mathrm{p}}$. If higher resolution CER measurements could be obtained, then AELM and PELM could be determined experimentally. As an example of the application of the MIST ELM model, Figure 1 shows the evolution of the neon density in DIII-D (shot 86944) in which neon was continuously injected during the discharge, thereby producing a change in $\mathrm{fELM}_{\mathrm{EL}}$ from $55 \mathrm{~Hz}$ down to $10 \mathrm{~Hz}$ starting at $\mathrm{t} \sim 2.75 \mathrm{~s}$. With the ELM model, the Ne XI density measured by CER during this transition is fit with a time-independent core anomalous diffusivity $\left(D_{A}\right)$ and with $\rho_{E L M}=0.8$, while $f_{E L M}$ is determined from $D_{\alpha}$, and $A_{E L M}$ is selected for each ELM event between 0.3 and 0.6 . Figure 1(a) shows results from the model when the assumed ELM amplitude (neon expulsion fraction) is varied from $20 \%$ to $100 \%$ and $D_{A}=0.5 \mathrm{~m}^{2} / \mathrm{s}$; likewise, Fig. 1(b) shows a variation of the anomalous diffusivity (edge $D_{A}$ ) from 0.5 to $1.0 \mathrm{~m}^{2} / \mathrm{s}$ at a fixed ELM amplitude. Without the ELM model the transition must be modeled by varying $D_{A}$, while the fit in Fig. 1 was obtained with a fixed $D_{A}$ profile (edge value $=0.7 \mathrm{~m}^{2} / \mathrm{s}$ ). Best fits are obtained using a pinch coefficient, $C_{V}=0$, or pinch velocity, $V_{p}=0$, in contrast to earlier results for helium, in which $C_{V}=1$ was found to be optimum. The anomalous diffusivities which result from the comparison are typically a factor of 3 lower than the diffusivities inferred from the conventional time-averaged analysis, which includes ELM effects as part of the anomalous diffusivity. For core 
modeling, the effective global recycling coefficient is taken to be 0.96 for neon, as determined by fits to the pumping decay after impurity injection.

For comparison, the ELM model has been applied to model type-I ELM behavior in JET helium and neon exhaust experiments for the Mkl configuration. In Fig. 2a-c the time dependence of $D_{\alpha}$, carbon, and beryllium edge impurity light is shown for a typical helium case without argon frosting of the cryopump (pulse 30725). The strike point recycling light for each of the impurities shown in Fig. 2a-c exhibits a similar behavior. These regular type I ELMs exhibit a cyclic behavior which allows us to analyze intra-ELM behavior. The $D_{\alpha}$, carbon, and edge helium signals are shown in Fig. 2d-f where the individual ELM events have each been remapped onto a single characteristic cycle time, which starts with the beginning of the ELM event. While the $D_{\alpha}$ and impurity signals are correlated, the response to ELM events differs, reflecting the differences in recycling properties of the species. The carbon and beryllium behavior differs from that of the extrinsic (helium or neon) impurities and this allows us to use MIST modeling of the carbon and beryllium time dependence to establish the ELM-model parameters AELM and PELM. These ELM parameters are then used for the simulation of the extrinsic injected impurities, either helium or neon. Figure 3(a) compares the evolution of the helium density measured via charge exchange recombination spectroscopy (CER) with (solid line) and without (dashed line) the model for ELM impurity expulsion in JET (shot 30725), while Fig. 3(b) shows the neon evolution compared with the MIST ELM model (shot 32778). For both helium and neon, best fits are obtained using a pinch coefficient, $C_{V}=0$. The edge diffusivitiy which results from the MIST ELM modeling yields $D_{A}=1.2 \mathrm{~m}^{2} / \mathrm{s}$ with $A_{E L M} \approx 0.5$ and $\rho_{E L M}=0.8$; whereas, a value of $D_{A} \approx 4 \mathrm{~m}^{2} / \mathrm{s}$ is needed to describe the helium density evolution found in Fig. 3(a) if the ELM model is not used. From the modeling of these helium and neon experiments on DIII-D and JET, the value of DA which is obtained by explicitly treating the ELMs, is typically a factor of -3 lower than the diffusivities inferred from the conventional time-averaged analysis. For both DII-D and JET, utilizing this ELM MIST model a time independent $D_{A}=1.0 \mathrm{~m}^{2} / \mathrm{s}$ describes the data and no inward pinch term is required. These smaller values for $D_{A}$ are more in line with those expected from neoclassical theory, but a more detailed accounting of the core transport (comparison with neoclassical rates), SOL transport (with ELM effects), and divertor plenum impurity balance would also require simultaneous high time-and space-resolution CER measurements of the significant impurity components along with divertor plenum impurity concentrations, which currently are not available. The analysis presented here provides a direct and simple model for treating the ELM expulsion of impurities and provides a useful method for evaluating impurity transport in ELMing H-mode discharges. 


\section{Divertor enrichment}

The retention of impurities, $i$, (helium or neon) in the divertor is often characterized by the divertor enrichment, $\eta_{D i v}^{l}$, and the divertor compression, $C_{D i v}^{l}$ parameters. Here the divertor enrichment is defined as, the ratio of helium (neon) concentration at the midplane separatrix to the concentration at the divertor separatrix $\eta_{D i v}^{b} \equiv K_{i}($ div $) / K_{i}(m i d)$, and the divertor concentration $\left.K_{i}=n_{i} / n_{e}\right)$. The divertor compression is given by $C_{D i v}^{l} \equiv n_{H e}($ div $) / n_{H e}($ mid $)$, where ${ }^{n} \mathrm{He}^{(\text {div })}$ is the helium density in the divertor and $n_{H e}(\mathrm{mid})$ is the helium density at the midplane separatrix. More efficient exhaust of neon observed in JET Mk1 experiments relative to helium is consistent with previous observations in DIII-D and ASDEX-Upgrade $[9,20]$. The pumping plenum concentrations were measured in the latter experiments via a modified Penning gauge, and higher neon compression was observed relative to helium. The JET Penning gauge system for helium and neon measurements in the sub-divertor was not yet installed during the discharges discussed here; therefore, the b2.5 code has been used to estimate the relative divertor concentrations for comparison with DIII-D. Experimental measurements of core plasma parameters, helium and neon density profiles from CER, as well as divertor measurements are utilized as input to b2.5. The b2.5 model is an enhanced version of the b2 code which treats the role of fast reflected particles and plenum-induced gas sources. The particle and energy reflection coefficients derived from TRIM simulations [21] are used to determine the fraction of particles striking the divertor plate which undergo direct reflection $\left(R_{N}\right)$ and the fraction of the emitted energy which is carried by the reflected particles, RE. The remainder of the particles (unity recycling for non-argon frost cases) are emitted with thermal energies. The code has been run in 5 (deuterium and helium) and 13 (deuterium and neon) species versions. The STRAHL database [22] is used in the helium/neon comparisons.

Previous comparisons of helium enrichment [9] with that of other non-reacting gases (neon, argon) have explained the observed higher enrichment of neon (and argon) relative to helium by stressing the differences in ionization potential: a longer mean free path for recycled helium atoms leads to a first ionization outside the region of strong deuterium ionization near the divertor plate. Thus an increased $\mathrm{D}^{+}$flow in that region is ineffective in producing high enrichment. This finding led to the use of induced $\mathrm{D}^{+}$flows to enhance enrichment in DIII-D.

However, modeling of these JET MKl experiments suggests an equally important effect of fast reflected atoms in reducing the helium enrichment. JET Mk 1 experiments were conducted with beryllium coated carbon surfaces. Fig. 4 shows the variation in energy reflection coefficients (TRIM values) expected for helium and neon incident on carbon and beryllium for the energy range observed in these experiments [from divertor probes $\mathrm{T}_{\mathrm{e}} \approx 30$ to $60 \mathrm{eV}$, with corresponding impact energy $\left(3<Z>k T_{e}+2 T_{i} \leq \sim 200 \mathrm{eV}\right)$. Generally, smaller values are expected, due to the surface 
roughness of actual divertor plates and also due to the incomplete coverage of carbon with beryllium. Fig. 5 shows the results from the b2.5 calculations when the assumed energy reflection coefficient $\left(R_{E}\right)$ is varied with respect to the TRIM value for helium (conditions for pulse 33994) and neon (conditions for pulse 32778) by varying fTRIM from 0.1 to 1.0 through the relation $R_{E}$ $=\mathrm{f}_{\mathrm{TR} M} \bullet \mathrm{R}_{\mathrm{E}}(\mathrm{TRIM})$. From Fig. 5 the helium compression is -2 and for neon $\sim 12$ at the TRIM values ( $f_{T R I M}=1.0$ ), while the helium enrichment is -0.5 and for neon enrichment $\sim 1.1$. As the energy reflection coefficient is reduced for helium $\left(\mathrm{f}_{\mathrm{TRIM}}<1.0\right)$ the compression rises by a factor $\sim 5$, whereas, for neon the compression remains $\sim 12$. Because of the low value for the direct reflection coefficient $(\mathrm{R} \sim 0.01)$ for neon, most of the recycled neon emerges at thermal energies and with its larger mass the neon residence time in the vacuum plenum will be -300 times longer than that for deuterium. Some of the reported discrepancies $[9,23]$ in neon inventory during injection experiments may be due to this effect alone. Due to variations in the roughness of the divertor tiles $\mathrm{f}_{\mathrm{TRIM}}$ is probably between 0.5 to 1.0. Table I shows the deduced enrichment and compression values deduced for the JET Mkl divertor for helium and neon compared with those measured on DIII-D and ASDEX. Very good agreement is observed between the various experiments. Typically, the helium and neon enrichment are: $\eta_{D i v}^{H e} \approx 0.9, \quad \eta_{D i v}^{N e} \approx 1.1$, respectively; while the helium and neon compressions are found to be: $C_{D i v}^{H e} \approx 3$ and $C_{D i v}^{N e} \approx 11$, respectively. Values found in parentheses in Table I for DIII-D are enrichment and compression values with an induced scrape-off-layer flow [9] to intentionally increase the enrichment. Recent preliminary measurements [24] on the JET Mk2 divertor in L-mode indicate that the helium enrichment is $\sim 0.8$ to 1.1 , also providing confidence in the values reported here for Mk1 utilizing the B2.5 modeling.

\section{Conclusions}

Comparison of DIII-D and JET Mk1 helium and neon exhaust experiments has been made with respect to core transport (ELM effects) and divertor enrichment. The experiments are quite different due to significant differences in divertor geometry and the size of the tokamak. A comparative modeling study finds similar reductions in inferred edge diffusivity when an ELM instability model is applied. The ELM model replaces the usual ad hoc ELM-averaged diffusivity with new parameters describing the fraction of impurity density which is expelled during and ELM event and the radius over which the ELM is effective in purging impurities. For both DIII-D and JET, the edge diffusivity, $D_{A} \approx 4 \mathrm{~m}^{2} / \mathrm{s}$, is found if ELM effects are not included in the model; whereas, $D_{A} \approx 1.2 \mathrm{~m}^{2} / \mathrm{s}$ if the ELMs are explicitly included in the model without the need for a time varying $\mathrm{D}_{\mathrm{A}}$ or pinch velocity $\left(\mathrm{V}_{\mathrm{p}}=0\right)$. Since the inferred core radial diffusivities are reduced with respect to ELM-averaged models, a closer comparison with neoclassical theory for the diffusivity may be warranted. However, before this can be accomplished, there still remains the question of edge 
boundary conditions for use in such an analysis. A better comparison with neoclassical levels requires simultaneous high time- and space-resolution CER measurements of the significant impurity components along with detailed data for edge boundary conditions during ELMs. Utilizing the B2.5 code and experimentally measured core and divertor plasma properties, the divertor enrichment and compression in the JET Mk1 configuration were calculated and found to be sensitive to the differences in particle reflectivity between helium and neon. The much lower particle reflection coefficients for neon leads to a larger predicted enrichment, relative to helium. This is consistent with observations that neon is more readily exhausted than helium. Finally, a detailed examination of core transport, exhaust, and divertor enrichment should include the effect of impurity purging during ELM events, which are quite complex. To further investigate the fast time scales of ELM events and the underlying mechanisms for impurity entrainment and compression higher time resolution edge and divertor diagnostics will be required.

\section{References}

[1] J. Hogan et al., J. Nucl. Mater. 241 (1997) 68.

[2] E. Gauthier et al., in Proc. 24th EPS Conference on Controlled Fusion and Plasma Physics, Berchtesgaden, Germany, 1997, Vol. 21A, Part 1, p. 61.

[3] S. Clement et al., J. Nucl. Mater. (this conference) (1998)

[4] M. R. Wade et al., Phys. Review Letters 74 (1995) 2702.

[5] M. G. v. Hellermann et al., in Proc. 22nd Europ. Conf. on Controlled Fusion and Plasma Physics, Bournemouth, U.K., 1995, Vol. 19C, Part II, p. 012.

[6] P. J. Harbour et al., in Proc. 22nd Europ. Conf. on Controlled Fusion and Plasma Physics, Bournemouth, U.K., 1995, Vol. 19C, Part IV, p. 465.

[7] D. Reiter et al., Nuclear Fusion 30 (1990) 2141.

[8] G. Saibene et al., in Proc. 22nd Europ. Conf. on Controlled Fusion and Plasma Physics, Bournemouth, U.K., 1995, Vol. 19C, Part II, p. 121.

[9] M. Wade et al., Nuclear Fusion in press (1998)

[10] R. A. Hulse Nuclear Technology/Fusion 3 (1983) 259.

[11] R. Maingi et al., Nuclear Fusion 34 (1994) 283.

[12] B. J. Braams, in NET Report: EUR-FU/XII-80/87/68 (1987). 
[13] R. Schneider et al., J. Nucl. Mater. 196-198 (1992) 810.

[14] D. Reiter, in Forschungszentrum, Juelich: Report Juel-2599 (1992).

[15] L. Lauro-Taroni et al., Contributions to Plasma Physics 38 (1998) 241.

[16] M. Fichtmueller et al., J. Nucl. Mater. (1998) (this conference).

[17] J. Hogan et al., in Proc. 24th European Physical Society Conference on Controlled Fusion and Plasma Physics, Berchtesgaden, Germany, 1997, Vol. 21A, p. 1133.

[18] R. Dux et al., Plas. Phys. Contr. Fusion 38 (1996) 989.

[19] M. O'Mullane et al., in Proc. 22nd EPS Conference on Controlled Fusion and Plasma Physics, Bournemouth, U.K., 1995, Vol. III, p. 121.

[20] H. S. Bosch et al., J. Nucl. Mater. 241-243 (1997) 82.

[21] W. Eckstein, in IPP-Garching Report: IPP 9/217 (1998).

[22] K. Lackner et al., Z. Naturforsch. 37a (1982) 931.

[23] H.-S. Bosch et al., Plasma Phys. Control. Fusion 39 (1997) 1771.

[24] M. Groth et al., in Proc. 25nd Europ. Conf. on Controlled Fusion and Plasma Physics, 1998 (to be published).

[25] M. R. Wade et al., J. Nucl. Mater. (1998) (this conference). 
Table I: Comparison of Helium and Neon Enrichment and Compression factors in ELMing H-mode discharges for JET(Mk1), JET(Mk2), DIII-D and ASDEX-U.

\begin{tabular}{|c|c|c|c|c|}
\hline Impurity & a JET(Mk1) & JET(Mk2) [24] & DIII-D[9,25] & ASDEX-U[20,23] \\
\hline \hline $\begin{array}{c}\text { Helium } \\
\text { Enrichment }\end{array}$ & $0.6-1.0$ & $0.8-1.1$ & $\begin{array}{c}0.9 \\
(1.1)^{\mathrm{b}}\end{array}$ & $0.3-.4$ \\
\hline Neon Enrichment & 1.1 & & $\begin{array}{c}1.0-1.2 \\
(1.6-2.3)^{\mathrm{b}}\end{array}$ & \\
\hline Helium & $2-4$ & & $\begin{array}{c}4.3 \\
(6.1)^{\mathrm{b}}\end{array}$ & $\begin{array}{c}3.5 \\
1-3\end{array}$ \\
\hline Compression & & & $\begin{array}{c}4.6-7.8 \\
(6.2-14.2)^{\mathrm{b}}\end{array}$ & 13 \\
\hline Neon & 11 & & & \\
Compression & & & & \\
\hline
\end{tabular}

a values based upon b2.5 modeling described in the text.

b values found in experiments with induced flow in the scrape-off layer (see Ref. 7 and 24). 


\section{Figure Captions}

Fig. 1. Comparison of results from MIST ELM model (solid lines) with measured NeXI density(data points) versus time for DIII-D shot 86944. During the shot the ELM frequency varies from $55 \mathrm{~Hz}$ to $10 \mathrm{~Hz}$. (a) A variation of the assumed expulsion fraction due to ELMs in the model with $D_{A}=0.75 \mathrm{~m}^{2} / \mathrm{s}$ and $\rho_{E L M}=0.8$. (b) $A$ variation of $D_{A}$ is shown with expulsion fraction $=0.2$ and $\rho_{E L M}=0.8$. The inset in (a) shows a typical fit to the neon density radial profile.

Fig. 2. Measured strike point light for (a) $D_{\alpha}$, (a) carbon, (b) beryllium, and (c) helium for JET pulse 30725. The repetitive behavior during giant ELMs allows the depiction of the cyclic evolution of the same quantities, starting with an ELM event. (d) shows the $D_{\alpha}$ behavior for the four ELM cycles in (a), (e) shows the evolution of carbon during this cycle (based on (a), and (f) shows the same for helium (based on $\mathrm{c}$ ).

Fig. 3. (a) Comparison of MIST evolution with (solid line) and without (dashed line) the ELM model for helium injection in JET pulse 30725, without argon frost. $D_{A}=1.2 \mathrm{~m}^{2} / \mathrm{s}$ for solid curve and $D_{A}=3.6 \mathrm{~m}^{2} / \mathrm{s}$ for the dashed curve. (b) Comparison of MIST ELM model (solid line) for neon injection in JET pulse 35402 with $4 \mathrm{~Hz}$ giant ELMs. $\mathrm{D}_{\mathrm{A}}=$ $1.2 \mathrm{~m}^{2} / \mathrm{s}$ for the solid curve. The insert shows the ELM activity.

Fig. 4. Comparison of the energy reflection coefficients for deuterium, helium, and neon incident on carbon and beryllium for an assumed incidence angle of $45^{\circ}$. While deuterium and helium have similar values, energy reflection for neon is at least 10 times lower than either of these. The pulses considered here were conducted with both beryllium coated carbon tiles and beryllium tiles.

Fig. 5. Results of a b2.5 survey for conditions of JET pulse 33994 (helium) and 32778 (neon) showing the sensitivity of divertor and midplane helium (neon) concentrations, enrichment, and compression to the assumed fraction of the TRIM value, fRIM (see text). 

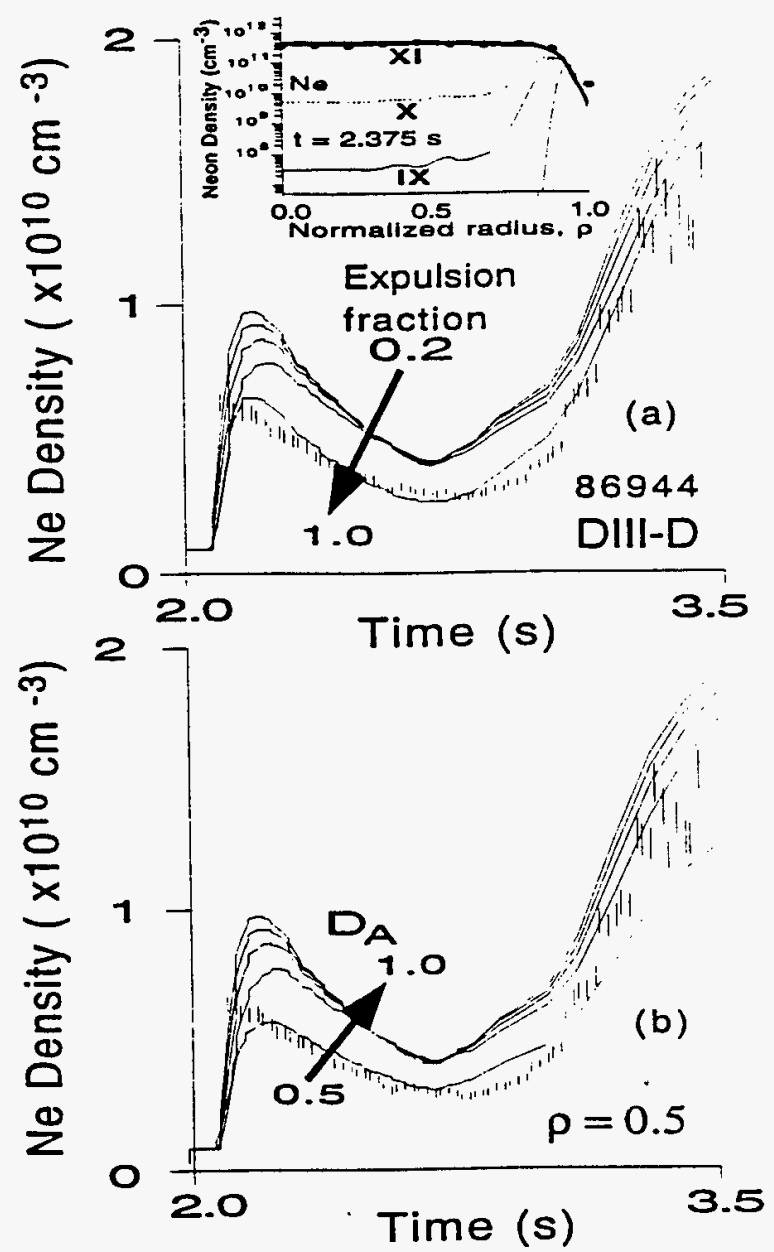

Fig. 1. Comparison of results from MIST ELM model (solid lines) with measured NeXI density (data points) versus time for DIII-D shot 86944 . During the shot the ELM frequency varies from $55 \mathrm{~Hz}$ to 10 Hz. (a) A variation of the assumed expuision fraction due to ELMs in the model with $D_{A}=0.75 \mathrm{~m}^{2} / \mathrm{s}$ and PELM $=0.8$. (b) $A$ variation of $D_{A}$ is shown with expulsion fraction $=0.2$ and $P E L M=0.8$. The inset in (a) shows a typical fit to the neon density radial profile. 

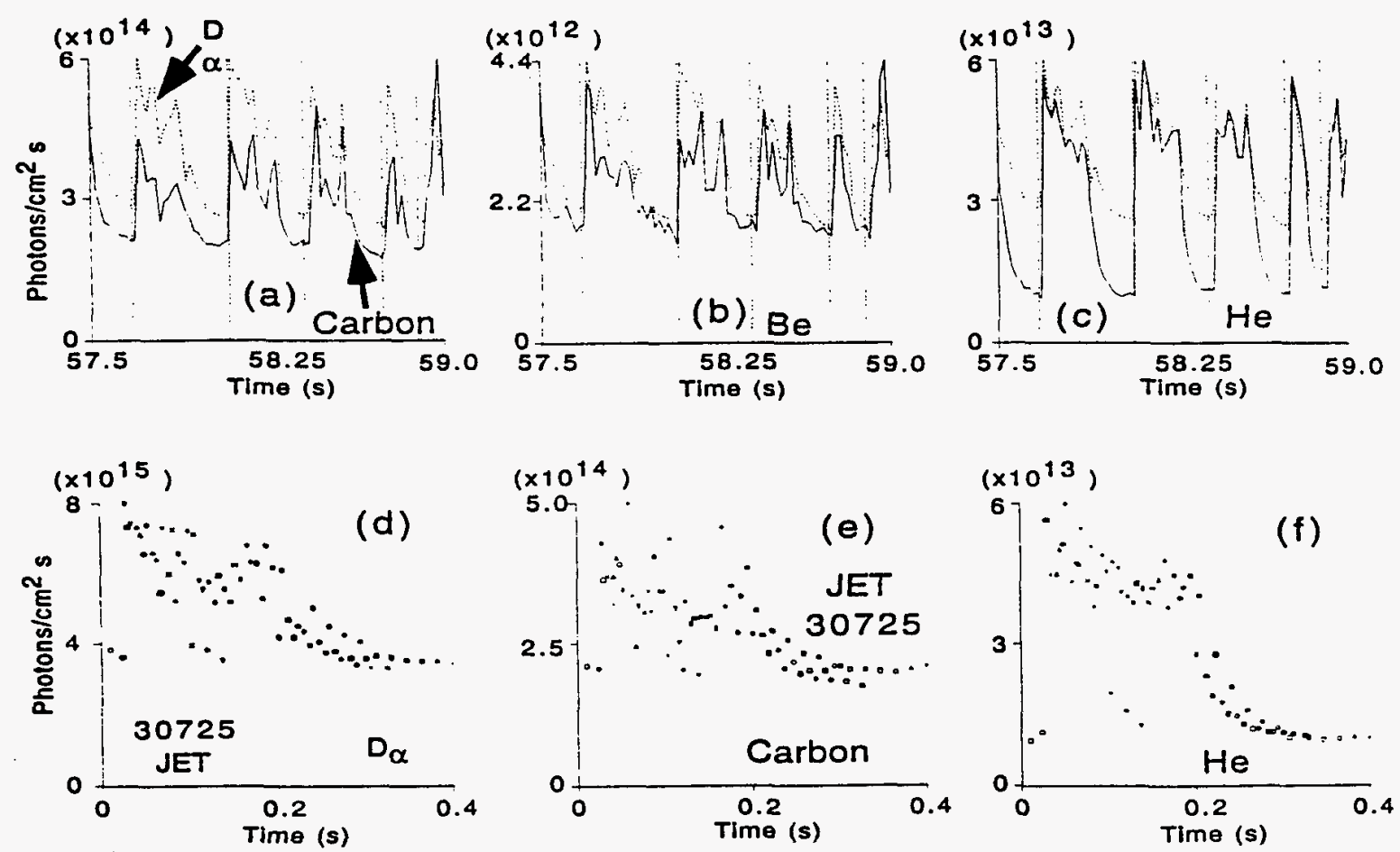

Fig. 2. Measured strike point light for (a) $D_{\alpha}$, (a) carbon, (b) beryilium, and (c) helium for JET pulse 30725. The repetitive behavior during giant ELMs allows the depiction of the cyclic evolution of the same quantities, starting with an ELM event. (d) shows the $D_{\alpha}$ behavior for the four ELM cycles in (a), (e) shows the evolution of carbon during this cycle (based on a), and (f) shows the same for helium(based on c). 


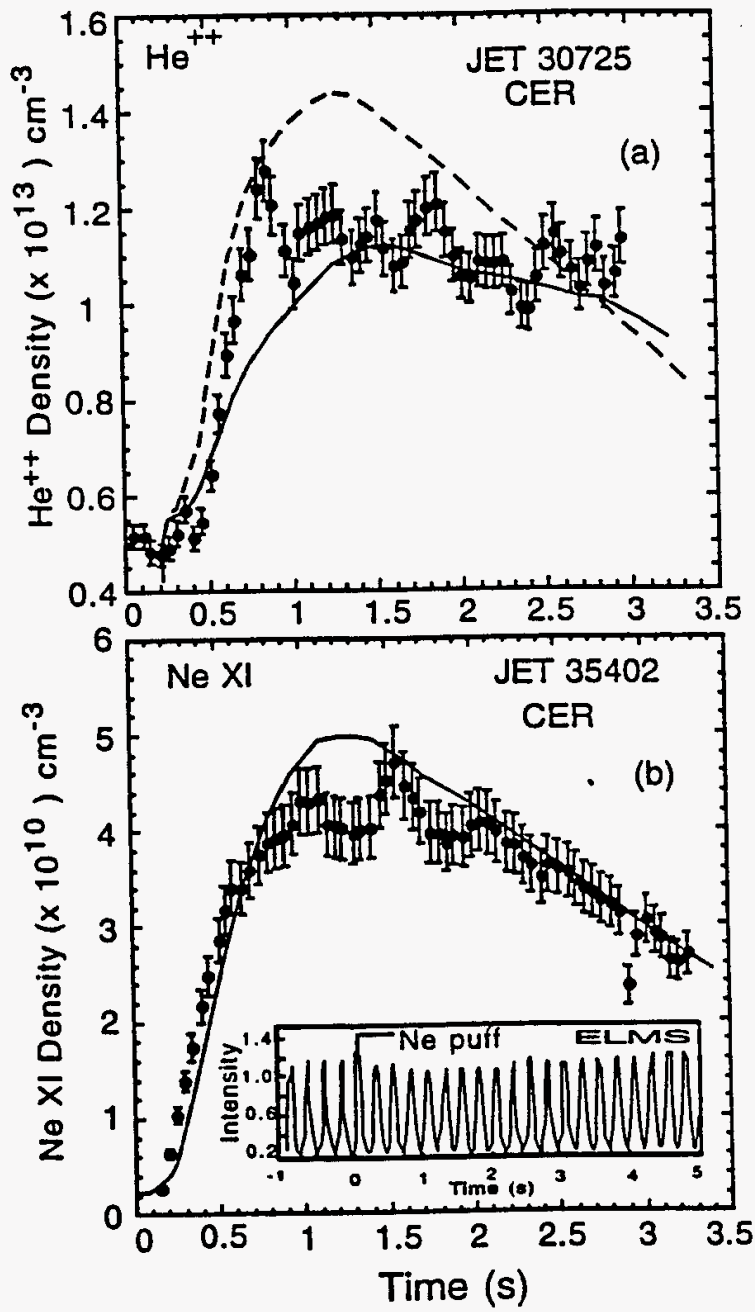

Fig. 3. (a) Comparison of MIST evolution with (solid line) and without (dashed line) the ELM model for helium injection in JET pulse 30725, without argon frost. $D_{A}=1.2 \mathrm{~m}^{2} / \mathrm{s}$ for solid curve and $D_{A}=$ $3.6 \mathrm{~m}^{2} / \mathrm{s}$ for the dashed curve. (b) Comparison of MIST ELM model(solid line) for neon injection in JET pulse 35402 with $4 \mathrm{~Hz}$ giant ELMs. $\mathrm{D}_{\mathrm{A}}=1.2$ $\mathrm{m}^{2} / \mathrm{s}$ for the solid curve. The insert shows the ELM activity. 


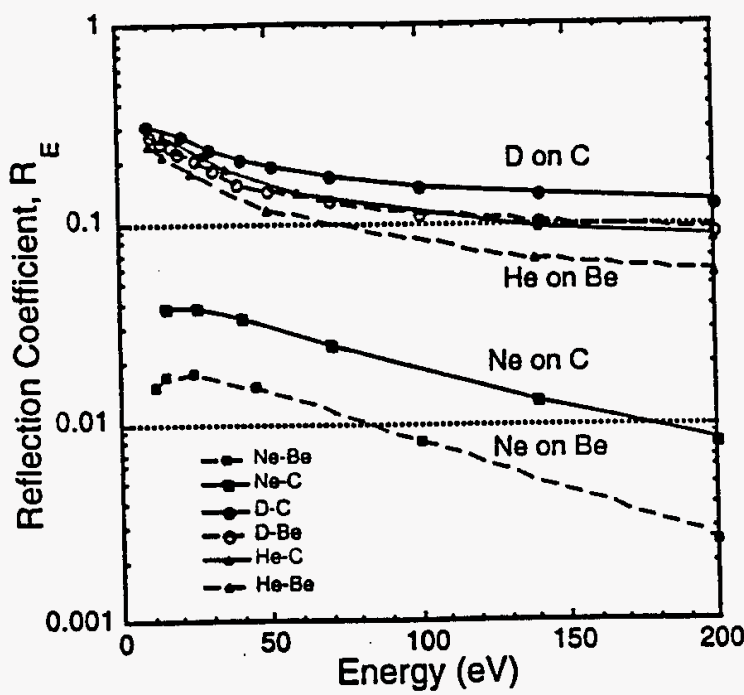

Fig. 4. Comparison of the energy reflection coefficients for deuterium, helium, and neon incident on carbon and beryllium for an assumed incidence angle of $45^{\circ}$. While deuterium and helium have similar values, energy reflection for neon is at least 10 times lower than either of these. The pulses considered here were conducted with both beryllium coated carbon tiles and beryllium tiles. 

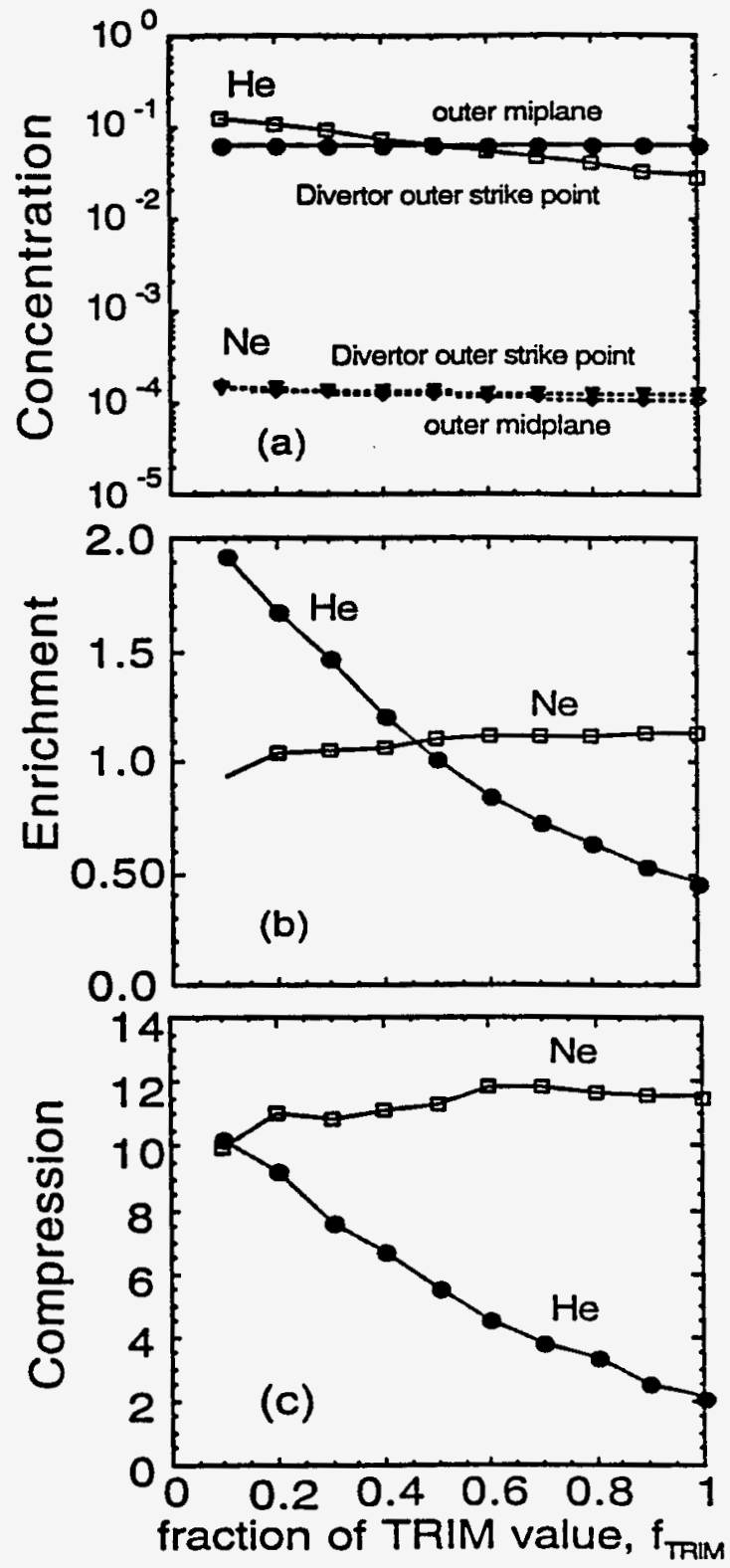

Fig. 5. Results of a b2.5 survey for conditions of JET pulse 33994 ( helium) and 32778 ( neon) showing the sensitivity of divertor and midplane helium (neon) concentrations, enrichment, and compression to the assumed fraction of the TRIM value, fTRIM (see text). 


\section{M98005577}

||||||||||||||||||||||||||||||||||||||||||||||||||||||||||||||||||

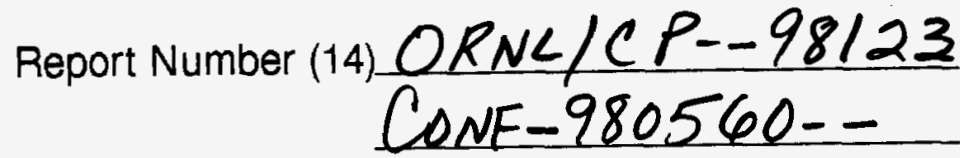

Publ. Date (11)

199805

Sponsor Code (18) DOE/ER, XF
UC Category (19) UC-400,DOE/ER 